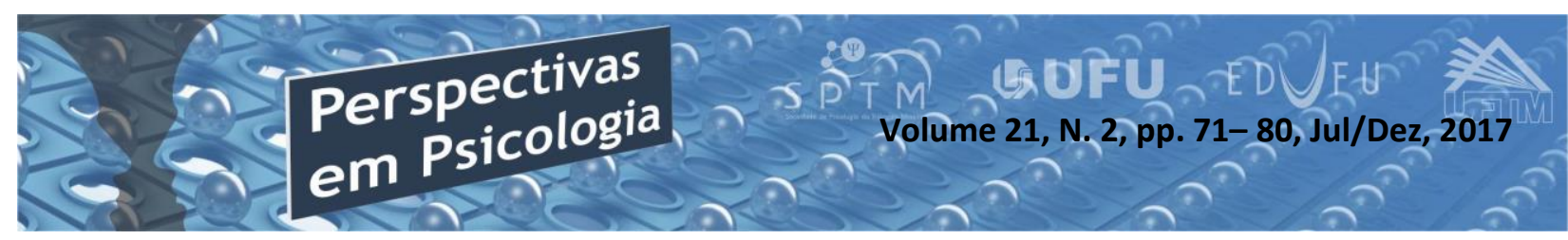

\title{
PERCEPÇÃO SOBRE OS FATORES ASSOCIADOS AO GANHO DE PESO EM PESSOAS QUE VIVEM COM HIV
}

\author{
Fernanda Gomes Pinhal \\ Cléria Maria Lobo Bittar \\ (Universidade de Franca - UNIFRAN - Franca - SP)
}

\begin{abstract}
Resumo
Introdução. Pessoas que vivem com o vírus do HIV (PV/HIV) além de lidaram com o preconceito tem lidado com novo fenômeno que é o excesso de peso. Estudos têm indicados que a superproteção da família e o tratamento com Terapias Antirretrovirais (TARV) podem estar associados a este aumento de peso. Objetivo. Conhecer a percepção de um grupo de pessoas com HIV sobre os fatores por eles alegado que expliquem o ganho de peso. Metodologia. Estudo transversal, quanti-qualitativo, com 58 participantes que apresentavam excesso de peso. Foi realizada entrevista semiestruturada visando à coleta individual de dados gerais e a percepção dos participantes sobre o ganho de peso. Resultados. O ganho de peso foi de até 75 quilos e os fatores associados a este foram ansiedade, perdas e luto, raiva e inconformismo. Conclusão. O consumo alimentar inadequado foi desencadeado por fatores psicológicos que refletiram diretamente no ganho de peso e estado nutricional das PV/HIV.
\end{abstract}

Palavras-chave: HIV; excesso de peso; fatores psicológicos.

\begin{abstract}
Perception About Factors Associated with Weight Gain in Persons Living with HIV
\end{abstract}

Introduction. People living with HIV (PL/HIV) in addition to dealing with prejudice have dealt with new phenomenon that is overweight. Studies have indicated that overprotection of the family and treatment with Antiretroviral Therapies (ART) may be associated with this weight gain. Goal. Know the perception of a group of people with HIV about the factors they alleged that explain the weight gain. Methodology. Cross-sectional, quantitative-qualitative study with 58 participants who were overweight. A semi-structured interview was conducted aiming at the individual collection of general data and the participants' perception of weight gain. Results. The weight gain was up to 75 kilos and the factors associated with this were anxiety, loss and mourning, anger and nonconformity. Conclusion. Inadequate food intake was triggered by psychological factors that directly reflected the PL/HIV' weight gain and nutritional status.

Key-words: HIV; overweight; psychology factors. 


\section{Introdução}

O quadro nutricional da população brasileira vem sofrendo mudanças de padrão, indicando coexistência entre desnutrição e excesso de peso em diversos segmentos da população. Este se apresenta com extrema gravidade, desequilibrando e colocando em risco a saúde do indivíduo, acarretando em comorbidades como hipertensão, doenças cardiovasculares, dislipidemias e diabetes (Brasil, 2004).

Segundo o VIGITEL- (Vigilância de fatores de risco e proteção para doenças crônicas por inquérito telefônico), 52,5\% dos brasileiros está acima do peso, o que caracteriza grave problema de saúde pública (Brasil, 2014).

Fatores fisiológicos, psicológicos, genéticos, ambientais, sedentarismo, baixa qualidade da dieta e a ingestão indiscriminada de alimentos industrializados, estão entre os fatores associados ao aumento de excesso de peso da população geral, mas alguns autores acreditam que os fatores psicológicos, estão entre os principais desencadeantes do ganho de peso (Abeso, 2001; Brasil, 2006; Quaioti \& Almeida, 2006; Wanderley \& Ferreira, 2010; WHO, 1998).

Entre os fatores psicológicos associados ao ganho de peso destacam-se: ansiedade, desenvolvimento emocional, percepção e controle sobre si mesmo, sensação de desamparo, insegurança e culpa. É ainda uma característica comum o comportamento de passividade e submissão, a ingestão compulsiva de alimentos e até mesmo drogas, a não aceitação do esquema corporal, a dificuldade de adaptação cultural e em lidar com frustrações (Wanderley \& Ferreira, 2010).

Dados do VIGITEL de 2014 mostram ainda que o excesso de peso é maior entre os homens e a maior prevalência está entre a população de 35 a 64 anos (Brasil, 2014). Tais dados se equiparam aos da população que vive com HIV, antes caracterizada apenas pela desnutrição e caquexia.

O Tratamento com Antirretrovirais (TARV) de alta potência, possibilitou melhorias na qualidade de vida dessas pessoas, incluindo o estado nutricional. Contudo Pessoas que Vivem com HIV (PV/HIV), vêm tendo que lidar com novas adversidades, entre elas o ganho/excesso de peso, que já acomete quase metade dessa população, destacando-se a prevalência entre o sexo masculino (Alencar et al., 2008; Rocha et al., 2013; Soares et al., 2015).

Pesquisas relatam possíveis fatores associados ao ganho/excesso de peso em PV/HIV, sendo eles muito parecidos com os 
fatores associados à população geral, como os históricos, ambientais, socioeconômicos, psicossociais, biológicos e culturais. Acrescentam-se também a estes fatores, o desejo de não parecer magro demais (aparentar o estereótipo da doença e sofrer possível preconceito), o uso contínuo de TARV e a influência e superproteção da família, não há, portanto, um consenso sobre os fatores associados ao excesso de peso nessa população (Jaime et al., 2006; Quaioti \& Almeida, 2006; Crum-Cianflone et al., 2008; Falco et al., 2012).

O objetivo desta investigação foi conhecer a percepção das PV/HIV sobre os fatores associados ao ganho de peso nesta população.

\section{Metodologia}

Trata-se de um estudo de corte transversal, quanti-qualitativo, realizado em unidade do Serviço de Atendimento Especializado em DST/Aids (SAE), do interior de São Paulo, no primeiro semestre de 2015. Para tal, foi utilizada uma entrevista semiestruturada, que visou à coleta individual de dados gerais, antropométricos e de percepção dos pacientes sobre o excesso de peso.

Participaram da pesquisa 58 pessoas que apresentavam excesso de peso, sendo 39 homens e 19 mulheres, adultos (entre 19 e 73 anos) com diagnóstico em HIV e assintomáticos. Foram coletados dados gerais dos participantes como idade, escolaridade, tempo de diagnóstico e uso de TARV, uso de álcool e drogas, prática de atividade física e presença de lipodistrofia, e medidas antropométricas para cálculo do IMC. Tal cálculo foi realizado com auxílio de calculadora virtual online oferecida pelo site do Ministério da Saúde.

Após coleta dos dados gerais e antropométricos, os 58 participantes que apresentaram excesso de peso, ou seja, IMC igual ou acima de 25, responderam à questão "O que você acha que ocorreu ou interferiu em sua vida, após o diagnóstico de HIV que justifique o ganho de peso?", e posteriormente responderam a um instrumento elaborado pelas pesquisadoras, cujo intuito era avaliar os possíveis fatores associados ao excesso de peso. A este instrumento, uma escala tipo likert, deu-se o nome Escala sobre Fatores Associados ao Ganho de Peso (EFAGP). O instrumento visava obter a percepção do próprio participante sobre os fatores que acarretaram no aumento de peso.

A EFAGP continha cinco questões que levaram em consideração os fatores associados ao excesso de peso em PV/HIV apontados pela literatura: 1) fatores sociais; 2) fatores psicológicos; 3) estigma da doença; 4) medicamentos; e 5) alimentação (Jaime et al., 2006; Quaioti \& Almeida, 
2006; Crum-Cianflone et al., 2008; Falco et al., 2012).

As respostas de cada questão da EFAGP variavam de 0 a 10 e foram categorizadas da seguinte maneira: de 0 a 3 referia-se a não interfere nada; de 4 a 7 interfere moderadamente; e de 8 a 10 interfere completamente.

A análise de dados foi realizada na versão 15 do programa SPSS (Statistical Package for the Social Sciences). No que tange às variáveis contínuas, os dados foram apresentados em média e desvio padrão. Para as variáveis categóricas, frequências e porcentagens foram descritas.

Para a análise das respostas à pergunta "O que você acha que ocorreu ou interferiu em sua vida, após o diagnóstico de HIV que justifique o ganho de peso?", foi realizado análise de conteúdo seguindo as orientações de Minayo (2010, 2012), como de compreender, interpretar e dialetizar, exercitar a interpretação de segunda ordem e produzir um texto fiel aos achados do campo, assegurando os critérios de fidedignidade e validade (Minayo, 2010; 2012).
O projeto foi submetido ao comitê de ética da Universidade de Franca, de acordo com a resolução 466/12, sob CAAE: 51114015.9.0000.5495. Também foi requisitada a autorização da Secretaria da Saúde, sendo iniciada a coleta de dados após a aprovação de ambos.

\section{Resultados}

Dos 58 participantes, $65,5 \%$ eram do sexo masculino e $34,5 \%$ do sexo feminino. A idade média foi de 46,6 anos $(\mathrm{DP}=12,2 \%)$.

Pôde-se perceber que o ganho de peso após o diagnóstico do HIV foi de até $75,0 \mathrm{~kg}$ e apenas duas pessoas relataram não ter sofrido variação de peso.

Ao categorizar as questões da EFAGP foi possível observar que a alimentação interfere de alguma forma para quase todos participantes $(86,2 \%)$, assim como os fatores psicológicos $(82,8 \%)$. Aqui é importante salientar que a EFAGP se refere à percepção dos participantes sobre os motivos que levaram ao aumento de peso. 
Tabela 1 - Categorização dos fatores associados ao excesso de peso em pessoas que vivem com HIV. (SAE - Franca, 2016).

\begin{tabular}{|c|c|c|c|}
\hline & $\begin{array}{l}\text { Não interfere } \\
\text { nada } \\
(\%)\end{array}$ & $\begin{array}{l}\text { Interfere } \\
\text { moderadamente } \\
(\%)\end{array}$ & $\begin{array}{l}\text { Interfere } \\
\text { completamente } \\
(\%)\end{array}$ \\
\hline Alimentação & 12,1 & 15,5 & 70,7 \\
\hline Fatores psicológicos & 15,5 & 34,5 & 48,3 \\
\hline Medicamentos & 32,8 & 24,1 & 41,4 \\
\hline Família e amigos & 72,4 & 20,7 & 5,2 \\
\hline Preconceito & 94,8 & 1,7 & 1,7 \\
\hline
\end{tabular}

No quadro 1 é possível visualizar as respostas de alguns participantes à questão "O que você acha que ocorreu ou interferiu em sua vida, após o diagnóstico de HIV que justifique o ganho de peso?" assim como os fatores psicológicos relacionados a elas.

Quadro 1 - Categorias de fatores psicológicos relacionados à alimentação.

\begin{tabular}{|c|c|}
\hline Categorias & Exemplos de relatos incluídos nas categorias \\
\hline \multirow[t]{2}{*}{ Ansiedade } & $\begin{array}{l}\text { Depende da minha ansiedade (...) desconto na comida, mas não sei de } \\
\text { onde vem tanta ansiedade, não (Mulher, } 49 \text { anos). }\end{array}$ \\
\hline & $\begin{array}{l}\text { Agora desempregado, fico ansioso e uso muitas drogas, aí dá aquela } \\
\text { larica, não tem jeito (Homem, } 58 \text { anos). }\end{array}$ \\
\hline \multirow[t]{2}{*}{ Depressão } & $\begin{array}{l}\text { Tive depressão pós-parto e ficava muito nervosa por que não conseguia } \\
\text { fazer nada na minha vida (...). Nem comer direito eu comia, era só sopa. } \\
\text { Comida eu vomitava. Mas ao invés de emagrecer, engordei (Mulher, } 40 \\
\text { anos). }\end{array}$ \\
\hline & $\begin{array}{l}\text { Foi muito impactante quando descobri a doença, cai na cama de tanta } \\
\text { depressão (...). Larguei futebol, descontava na alimentação. Pensava que } \\
\text { ia morrer (Homem, } 54 \text { anos). }\end{array}$ \\
\hline
\end{tabular}


PERCEPÇÃO SOBRE OS FATORES ASSOCIADOS AO GANHO DE PESO EM PESSOAS QUE VIVEM COM HIV

\begin{tabular}{|c|c|}
\hline \multirow[t]{2}{*}{ Raiva } & $\begin{array}{l}\text { Ele não aceita meu 'homossexualismo'(...) a raiva é tanta que tem dias } \\
\text { que desconto na comida (Homem, } 50 \text { anos). }\end{array}$ \\
\hline & $\begin{array}{l}\text { Eu tinha raiva do meu ex-marido (...). Quando ficava sozinha em casa só } \\
\text { pensava em comer tudo quanto é tipo de doces e salgadinhos (Mulher, } 58 \\
\text { anos). }\end{array}$ \\
\hline \multirow[t]{2}{*}{ Estresse } & $\begin{array}{l}\text { Depois da separação minha vida virou um inferno e pra piorar perdi o } \\
\text { emprego. A gente passa nervoso e desconta no prato (Homem, } 52 \text { anos). }\end{array}$ \\
\hline & $\begin{array}{l}\text { O estresse com o final da faculdade, com certeza! Como mal, como errado } \\
\text { e como muito (Homem, } 26 \text { anos). }\end{array}$ \\
\hline \multirow[t]{2}{*}{ Perdas e luto } & $\begin{array}{l}\text { Após a perda dos meus pais a comida passou a ser minha companheira, } \\
\text { principalmente a noite, como se fosse para suprir algo (...), me convidam } \\
\text { pra sair, vou só pra não ficar só (Mulher, } 61 \text { anos). }\end{array}$ \\
\hline & $\begin{array}{l}\text { Quando perdi o emprego vivia angustiado e a alimentação só piorou } \\
\text { (Homem, } 44 \text { anos). }\end{array}$ \\
\hline
\end{tabular}

\section{Discussão}

Para justificar o ganho de peso, todos os participantes expressaram alguns dos fatores psicológicos acima citados. No entanto, para apresentação nesta tabela, foram selecionadas apenas 10 das 58 falas.

O processo para a categorização das falas dos participantes consistiu em triagem dos conteúdos e agrupamento daqueles que apresentavam as mesmas palavras em seu contexto e/ou o mesmo sentido nas frases.

Com o presente estudo, observou-se uma mudança no estado nutricional e comportamento alimentar de PV/HIV que antes se caracterizavam pela caquexia e desnutrição. Tal mudança de estado nutricional já pôde ser observada em outros estudos, como os de Alencar et al. (2008), Jaime et al. (2006), Crum-cianflone et al. (2008), que também associaram a mudança 
no estado nutricional à idade, sexo e tempo de infecção pelo vírus.

Durante as entrevistas, foi possível observar que PV/HIV vêm tendo que lidar com adversidades oriundas da doença e com situações de cunho particular que também podem influenciar no estado psíquico e consequentemente, no estado nutricional destas pessoas.

Com a análise dos resultados notouse que o fator "alimentação" foi o principal fator associado ao excesso de peso, de acordo com a escala EFAGP. O fator "alimentação" foi citado por $86,2 \%$ dos entrevistados, seguido pelo fator "psicológico" citado por $82,8 \%$.

Entretanto, a partir da análise sobre a questão: "O que você acha que ocorreu ou interferiu em sua vida, após o diagnóstico de HIV que justifique o ganho de peso?", percebeu-se que em grande parte dos casos o fator "alimentação" era na realidade desencadeado pelo fator "psicológico".

Foi possível observar que o comportamento alimentar estava associado a eventos importantes da vida dos participantes e que fatores externos à doença também corroboraram para o mau comportamento alimentar, como sentimentos de raiva, estresse, ansiedade, entre outros.

Notou-se que estas pessoas ao lidarem com as situações cotidianas, geralmente associam-nas com as adversidades de se viver com o vírus HIV, ou seja, dificilmente consegue desvincularse da condição e do estigma da doença, o que acaba acarretando maior sofrimento para as mesmas.

Notou-se também que, muitos dos participantes escondiam da família/amigos a sua situação clinica, enquanto outros acabaram se afastando ou sendo afastados de seus grupos sociais. Por este comportamento foi possível identificar que o isolamento e solidão, por exemplo, também corroboraram para descompasso do comportamento alimentar.

A psicologia em diversas teorias versa sobre o comportamento alimentar como forma compensatória. Estudos de Caetano et al (2005) e Wanderley e Ferreira (2010) mostram que pessoas depressivas ou que estejam passando por momentos de ansiedade são mais suscetíveis a cair nesta armadilha e usar o alimento, principalmente os mais calóricos e menos nutritivos, como massas, doces e frituras, como forma de “descontar a tensão" (Caetano et al., 2005; Wanderley \& Ferreira., 2010).

Entre as PV/HIV isso se deve também ao impacto do diagnóstico e mudanças no estilo de vida, adaptações psicológicas, mudanças físicas relacionadas ao tratamento com antirretrovirais e seus efeitos colaterais, causando mudanças na autoestima, depressão e isolamento social (Espanha, 2015). 
Estudos de Bing et al. (2001) e Pence et al. (2006) mostram que transtornos de humor e ansiedade são comuns nesta população, que acaba tendo dificuldade em controlar o consumo de alimentos, principalmente em situações de tristeza, ansiedade, estresse, solidão e frustração, resultando num padrão contínuo de uso da alimentação como forma de lidar com suas emoções (Bing et al., 2001; Pence et al., 2006).

Pelo presente estudo ficou evidente que as relações afetivas (família e amigos) e processos psicossociais interferem diretamente no desenvolvimento das práticas de saúde da população em questão, e que o enfretamento da doença é uma situação estressante que pode desencadear respostas psíquicas importantes.

A alimentação é um comportamento derivado de diversos segmentos e envolve muito mais do que apenas a nutrição do corpo. O paladar, o preço, a facilidade no preparo e a publicidade condicionam as escolhas alimentares que realizamos. Vários aspectos como falta de tempo, ansiedade e dificuldade do consumo satisfatório de alimentos influenciam no estilo de vida e interações do sujeito com o meio em que vive, e todos estes fatores devem ser levados em consideração.

Contudo, conclui-se com esta pesquisa que o fator alimentação influenciou diretamente no ganho de peso da população estudada e o fator "psicológico" contribuiu de forma indireta. Desta forma, é possível inferir que eventos estressantes do cotidiano ou até mesmo as adversidades de se viver com uma doença sem cura como o HIV, pode refletir nas escolhas e hábitos alimentares, podendo acarretar em excesso de peso e outras comorbidades.

\section{Considerações Finais}

A discussão a cerca do tema proporcionou um olhar novo e diferente sobre a problemática em questão, possibilitando o conhecimento sobre a percepção das PV/HIV. Assim sendo, enfoca-se a importância de um acompanhamento multiprofissional que englobe as diversas necessidades da PV/HIV, incluindo a atenção a sua saúde nutricional e mental. A compreensão do fenômeno do ganho de peso em relação à população estudada contribui para o melhor desenvolvimento de políticas e ações tanto para a prevenção do fenômeno, quanto para promoção de ações visando à sua saúde.

A discussão sobre o HIV/Aids não deve, portanto, limitar-se apenas aos fatores físicos, mas deve também aprofundar-se em aspectos emocionais e comportamentais dos indivíduos. 


\section{Referências}

ABESO - Associação Brasileira para o Estudo da Obesidade e Síndrome Metabólica (2001). Documento do Consenso Latino-Americano em Obesidade. São Paulo.

Alencar, D. T. M., Nemes, M. I. B. \& Velloso, M. A. (2008). Transformações da "aids aguda" para a "aids crônica": percepção corporal e intervenções cirúrgicas entre pessoas vivendo com HIV e aids. Ciencia \& Saude Coletiva, 13 (6), 1841-1849. https://doi.org/10.1590/S1413-81232008000600019

Brasil (2004). Ministério da Saúde. Vigilância alimentar e nutricional, SISVAN. Brasília.

Brasil (2006). Ministério da Saúde. Secretaria de Atenção à Saúde. Departamento de Atenção Básica. Obesidade. Brasília.

Brasil (2014). Ministério da Saúde. Vigilância de fatores de risco e proteção para doenças crônicas por inquérito telefônico, VIGITEL. Brasília.

Bing, E. G., Burnam, M. A. \& Longshore, D. (2001). Psychiatric disorders and drug use among human immunodeficiency virus-infected adults in the United States. Arch Gen Psychiatry. 58, 721-728. https://doi.org/10.1001/archpsyc.58.8.721

Caetano, C., Carvalho, A. M. P. \& Galindo, E. M. C. (2005). Obesidade e aspectos psicológicos: maturidade emocional, auto-conceito, Locus de controle e ansiedade. Rev. Psicol. Reflex. Crit., 18 (1). https://doi.org/10.1590/S0102-79722005000100006

Crum-Cianflone, N., Tejidor, R., Medina, S., Barahona, I. \& Ganesan, A. (2008). Obesity among Patients with HIV: The Latest Epidemic. Aids Patient Care and Stds, 22(12), 925930. https://doi.org/10.1089/apc.2008.0082

Espanha (2015). Documento de consenso sobre las alteraciones psiquiatricas y psicológicas en adultos y niños con infección por el VIH. Madri.

Falco, M., Castro, A. D. C. D. O., \& Silveira, E. A. (2012). Nutritional therapy in metabolic changes in individuals with HIV/AIDS. Revista de Saude Publica,46(4), 737-746. changes in individuals with HIV/AIDS. Revista de Saude Publica,46(4), 737-746. https://doi.org/10.1590/S0034-89102012000400019

Jaime, P. C., Florindo, A. A., Latorre, M. D. R. D. D. O. \& Segurado, A. A. C. Obesidade abdominal e consumo alimentar em portadores de HIV/Aids. Revista De Saude Publica, 40(4), 634-640.

Minayo, M.C. de S. (2010). O desafio do conhecimento. São Paulo, Hucitec.

Minayo, M. C. de S. (2012). Análise qualitativa: teoria, passos e fidedignidade. Ciênc. saúde coletiva [online].17(3),621-626. Acesso 31/012017. Disponível em: $<$ http://www.scielo.br/scielo.php?script=sci_arttext\&pid=S1413 
$81232012000300007 \& \operatorname{lng}=$ en\&nrm=iso $>$.

8123. http://dx.doi.org/10.1590/S1413-81232012000300007.

Pence, B. W., Miller, W. C., Whetten, K., Eron, J. J. \& Gaynes, B. N. (2006). Prevalence of DSM-IV-defined mood, anxiety, and substance use disorders in an HIV clinic in the southeastern United States. Journal Acquir Immune Deficience Syndrome, 42, 298-306. https://doi.org/10.1097/01.qai.0000219773.82055.aa

Quaioti, T. C. B. \& Almeida, S. S. (2006). Determinantes psicobiológicos do comportamento alimentar: uma ênfase em fatores ambientais que contribuem para a obesidade. Psicologia USP, 17(4), 193-211. https://doi.org/10.1590/S0103-65642006000400011

Rocha, G. M. N., Wall, K. M., Peralta, M. C., Martinez, A. M. S. \& Torres, R. A. B. (2013). Atención nutricional, tiempo desde el diagnóstico, características demográficas e índice de masa corporal en pacientes con VIH/SIDA. Revista de Investigación Clínica, 65(4), 291299.

Soares, L. R., Silva, D. C., Gonsalez, C. R., Batista, F. G., Fonseca, L. A., Duarte, A. J. \& Casseb, J. (2015). Discordance between body mass index and anthropometric measurements among hiv-1-infected patients on antiretroviral therapy and with lipoatrophy/lipohypertrophy syndrome. Rev Inst Med Trop São Paulo, 57 (2), 105-110. https://doi.org/10.1590/S0036-46652015000200002

Wanderley, E. N. \& Ferreira, V.A. (2010). Obesidade: uma perspectiva plural. Ciênc. saúde coletiva, 15(1). https://doi.org/10.1590/S1413-81232010000100024

WHO-World Health Organization (1998). Obesity: Preventing and managing the global epidemic. Report of a WHO Consultation on Obesity.

Agradecimento à CAPES - Coordenação de Aperfeiçoamento de Pessoal de Ensino Superior, pela concessão da bolsa para a realização desta pesquisa.

\section{As autoras:}

Fernanda Cristina Gomes Pinhal possui graduação em Psicologia pelo Centro Universitário UNIFAFIBE. É mestre em Promoção de Saúde pela Universidade de Franca - UNIFRAN. Atualmente, doutoranda pelo mesmo programa. E-mail: nandapinhal@hotmail.com

Cléria Maria Lobo Bittar, graduação em Psicologia e Direito, ambos pela Universidade de Franca, mestrado e doutorado em Serviço Social pela Universidade Estadual Paulista Júlio de Mesquita Filho, Pós-Doutorado pelo Instituto de Estudios Universitarios de la Mujer, pela Universidade de Valência, Espanha. Docente permanente do Programa de Mestrado e Doutorado em Promoção da Saúde e do curso de Psicologia da Universidade de Franca. E-mail: profa.cleriabittar@gmail.com

Recebido em: 16/09/2017

Aprovado em: 27/12/2017 